\title{
LES PARALYSIES DES QUATRE DERNIERS NERFS CRANIENS ET DU SYMPATHIQUE CERVICAL: SYNDROME DE VILLARET
}

\author{
H. RogEr \\ C. Raybaud
}

Les paralysies des derniers nerfs craniens peuvent se grouper suivant trois modalités: (1) Ou bien l'atteinte porte uniquement sur les nerfs qui passent par le trou déchiré postérieur (IX, X, XI), realisant le syndrôme du trou déchiré postérieur décrit par Vernet. (2) Ou bien à cette atteinte des, IX, X, XI paires se surajoute celle de l'hypoglosse. C'est en Septembre 1914 que Collet observa chez un blessé de guerre un syndrôme caractérisé par l'hémiplégie du voile du palais, du larynx, du pharynx et de la langue, et qu'il désigna sous le nom de syndrôme total des quatre derniers nerfs; dès cette première observation il insistait sur l'importance de la dysphagie, particulièrement marquée pour la déglutition des solides. Sicard et Bollack, en 1912, avaient décrit sous le nom "d'hémiplégie linguale laryngo-pharyngienne et cléido-trapézienne avec tachycardie" un syndrôme très analogue avec accélération marquée du pouls, mais où la symptomatologie pharyngée était moins précisée. Ce syndrôme porte encore le nom de Collet et Sicard ou celui de "syndrôme du carrefour condylo-déchiré postérieur" en raison du trou déchiré postérieur par lequel passent les IX, X, XI paires, et du trou condylien qui donne passage au XII. Enfin, à cette paralysie des quatre derniers nerfs craniens peut se superposer celle du sympathique cervical supérieur, conme Villaret l'a montré en apportant, en 1916, une série d'observations d'un nouveau syndrôme auquel il donna le nom de "syndrôme de l'espace sous-parotidien postérieur" en raison de la région anatomique où sont groupés ces divers nerfs; on l'appelle depuis lors "syndrôme de Villaret".

C'est à ce dernier que nous avons l'intention de consacrer celte étude, à la lumière de nos observations personnelles, des cas recueillis dans la littérature, et de la monographie consacrée par Collet aux troubles de l'innervation pharyngo-laryngée et esophagienne (Masson et Cie., édit., Paris, 1946).

Avant d'envisager les diverses étiologies susceptibles de réaliser ce syndrôme nous voudrions rappeler la symptomatologie que réalise ce groupement de paralysies: La paralysie du IX-moteur se traduit, du point de vue fonctionnel, par des troubles de la déglutition très marqués; la déglutition des solides est très difficile, le bol alimentaire s'arrête à l'entrée du pharynx, et une gorgée d'eau est souvent nécessaire pour le faire cheminer plus loin. Du point de vue objectif elle se marque, surtout pendant la nausée que provoque le contact de l'abaisse-langue, par un mou- 
vement de translation de la paroi postérieure du pharynx vers le côté sain; l'hémi-pharynx paralysé est, en raisori de son inertie, entraîné du côté opposé par la contraction de l'hémi-pharynx sain (signe du rideau). Soulignons cependant que l'atteinte du $\mathrm{X}$ joue peut-être un rôle dans l'apparition de ces troubles pharyngés: la part respective du IX et du $\mathrm{X}$ dans l'innervation motrice du pharynx n'a pas encore été complètement élucidée. La lesion du IX-sensitif explique l'hypoesthésie au tact et à la piqûre de la partie postérieure de la langue et, surtout, les troubles du goût: agueusie du tiers postérieur de la langue, surtout marquée pour les substances amères, le IX étant leur principal agent de transmission. Pour certains physiologistes cependant, les deux-tiers antérieurs de la langue doivent aussi leur sensibilité gustative au IX, mais par voie anastomotique: l'abolition de la sensibilité sur toute une moitié de la langue pourrait, dans ce cas, indiquer une lésion du nerf à son origine ou dans son trajet intra-cranien, alors qu'une agueusie limitée au tiers postérieur correspondrait à une lésion extra-cranienne. La paralysie du X et XI (branche interne) est responsable de l'hémiplégie vélo-palatine, manifestée par une paralysie unilatérale typique du voile avec déviation de la luette pendant la phonation, et de l'hémiplégie laryngée, manifestée par des troubles de la voix et l'immobilité d'une corde vocale constatable au laryngoscope. L'atteinte du XI (branche externe) détermine l'hémiplégie scapulaire traduite par l'abaissement du moignon de l'épaule et la difficulté à le relever (trapèze), par la gêne dans le mouvement de rotation de la tête (sterno-cléido-mastoïdien), et l'atrophie relative $\mathrm{du}$ muscle, perceptible à la vue et au pincement. On peut également mettre en évidence cette paralysie dans l'acte de hausser les épaules, qui montre un creusement moins marqué du creux sus-claviculaire du côté malade. La paralysie $d u X I I$ détermine l'hémiplégie linguale se traduisant par l'hémiatrophie linguale et des contractions fibrillaires unilatérales ainsi que par la déviation de la langue (en forme de crochet) vers le côté paralysé, lorsque celle-ci est tirée hors de la bouche. La paralysie du sympathique cervical supérieur réalise le syndrôme de Claude Bernard-Horner avec myasis, rétrécissement de la fente palpébrale et énophtalmie, auxquels peuvent s'associer des troubles vaso-moteurs ou sudoraux de l'hémi-face correspondante.

Depuis la description initiale de Villaret, les observations de ce syndrôme si typique se sont multipliées et ont mis en évidence la diversité des causes qui peuvent en être responsables. On peut reconnaître au syndrôme de Villaret des causes traumatiques et des causes non traumatiques.

1) Causes traumatiques - Il peut s'agir de traumatisme par instrument piquant pénétrant profondément au-dessous du conduit auditif externe, en avant de la pointe de la mastoïde, en arrière de la branche montante du maxillaire inférieur ou il peut s'agir de fracture de la base du crâne (Courbon, Soc. d'Oto-Neuro-Ophtalmologie de Strasbourg, 10 Jan vier 1925 - Galand, J. Belge de Neurologie et Ps:chiâtrie, Octobre 1932), mais ces deux ordres de faits sont assez rares. Le plus souvent le syn- 
drôme est réalisé par une balle ou un éclat d'obus, et c'est en effet à propos de blessures de guerre que Collet, puis Villaret, ont décrit les syndrômes qui portent leur noin. Plusieurs observations de tels cas ont été relatées: Sicard et Roger, "Paralysie des quáatre derniers nerfs craniens avec association de lésions du sympathique cervical par éclat d'obus extracranien", Soc. Méd. Chir. de la XVème Région, 3 Oct. 19]8; Rimbaud et Vernet, "Syndrôme condylo-déchiré postérieur", Soc. Méd. Chir. XVème Région, 3 Oct. 1918; Bellin et Vernet, "Un cas de syndrôme du trou déchiré postérieur", ibid., 18 Juillet 1918; Worms et Lioux, "Paralysies des quatre dernières paires et du sympathique cervical par blessure de guerre", Soc. d'Oto-Neuro-Ophtalmologie de Paris, 4 Déc. 1925; Daraban, Société Roumaine d'Oto-Laryngologie, 12 Déc. 1930.

2) Causes non traumatiques - Les néoplasies et les suppurations occupent la première place. On a décrit des cas de tumeurs de la parotide à l'origine du syndrôme de Villaret: Canuyt et Klotz, Soc. d'Oto-NeuroOphtalmologie de Strasbourg, 14 Déc. 1928; Rimbaud, Terracol et Chardonneau, Soc. d'Oto-Neuro-Ophtalmologie du Sud-Est-Montpellier, Oct. 1928. Collet et Bonnet avaient mis en évidence, en 1923, dans les squirrhes de la parotide un syndrôme paralytique caractérisé par l'adjonction d'une paralysie faciale au syndrôme de Villaret. C'est, quelquefois, une tumeur de la base du crâne (Vasiliu-Grigoresco et Brull, Soc. d'Oto-NeuroOphtalmologie de Bucarest, 23 Juin 1930; G. Cosia-Elice, "Multiple paralysis of cranial nerves and tumours of neso-pharynx", Arch. Neurol. a. Psychiat., Sept. 1948; Varneau, Revue de Laryngologie, 31 Déc. 1924). Une adénopathie néoplasique peut aussi réaliser le syndrôme de Villaret (Halphen, Soc. de Laryngologie des Hôpitaux de Paris, 9 Fév. 1931).

Une étiologie assez rare est la suppuration de la mastoïde, frappant les cellules postéro-inférieures de cette apophyse (Lannois et Molinier, Lyon-Médical, Avril 1919; Fiocre, "Mastoïdite postérieure avec fusées purulentes sous-occipitales et syndrôme nerveux condylo-déchiré postérieur"). Pareilles suppurations viennent plutôt de l'amygdale, mais à notre connaissance à peine quelques cas en ont été publiés (Palumbo, Archives Italiennes d'Otologie, Oct. 1924; Bloc, Soc. Laryng. des Hôp. de Paris, 9 Mars 1927; Aloin Gaston et Yves Lemaître, Soc. Laryng. de Paris, 14 Mars 1932; Rouffart, Le Scalpel, 25 Janvier 1934). Le cas de Canuyt (Soc. Méd. et Chir. de Bordeaux, 9 Juillet 1920) nous paraît entrer dans ce cadre, parce que survenu après des phénomènes infectieux du type angineux, quoique l'auteur invoque une révrite vraisemblablement syphilitique (?) malgré un B.W. négatif. Quant au cas de Chabrol, Dufourmentel et Michel- "Une cause exceptionnelle de syndrôme rétro-parotidien: le phlegmon péri-amygdalien", Paris Médical, 12 Mars 1926), peut-être se prête-t-il à discussion, car il s'agit d'une paralysie bilatérale survenue comıme terminaison d'un état cachectique, pour lequel on peut se demander s'il ne s'agirait pas de lésion centrale bulbaire entraînant cette double parésie, en même temps qu'une parotidite phlegmoneuse. 
On a pu, enfin, retrouver à l'origine du syndrôme de Villaret une échinococcose, un anévrysme carotidien (Canuyt, Soc. Méd. dú Bas-Rhin, 27 Nov. 1921).

Des causes intra-craniennes et bulbaires sont susceptibles de provoquer des paralysies associées des quatre derniers nerfs craniens: tumeur de la dure-mère (Rabattu, Rev. Larying. 31 Oct. 1927); tumeur de l'étage postérieur du crâne (Sicard et Roger, Marseille Médical, 1929); anévrysme intra-cranien (Bailliart, Montuis e Schiff-Wertheimer, Bull. Soc. d'Ophtalmologie de Paris, Juin 1931); méningite localisée prebablement syphilitique (Denoyer, Minerva Medica, 19 Mai 1931). Mais il ne s'agit pas à proprement parler de syndrôme de Villaret, car il n'y a pas, en général, association de troubles pupillaires et de syndrôme de Claude Bernard-Horner. Il en est de même des lésions bulbaires dont le diagnostic se fait par les symptômes surajouiés signant l'origine cenirale. Telle une hémiplégie du côté opposé dans le cas de van Caneghen (Soc. Belge d'Otologie, 9 Juil., 1926), ou plus exceptionnellement du même côté (Sicard et Rimbaud, Paris-Médical, Sept., 1917); telle une paralysie Duchenne-Erb avec syndrôme de Claude Bernard-Horner et dissociation des sensibilités par syringobulbie dans un cas que l'un de nous a presenté avec Brémond (Société d'Oto-Neuro-Ophtalmologie du Sud-Est, 25 Fév., 1925); telle une thermo-anesthésie du membre supérieur et un nystagmus (Clovis Vincent et Winter, Soc. d'Oto-Neuro-Ophtalmologie de Paris, 4 Sept., 1925) ou une parésie passagère du plexus brachial dans le cas de Heindl (Soc. Viennoise de Laryngologie, 4 Janv., 1900).

Le cas de notre malade, que nous allons mairienant relater, entre dans le groupe des causes locales non traumatiques puisqu'il s'agit d'un abcès de l'amygdale suivi de phlegmon de la région cervicale.

Antoine Pié..., cultivateur corse de 54 ans présente, au début de Décembre 1950, un état fébrile avec tuméfaction latérale du cou et douleurs à la déglutition, s'irradiant vers la région sus-claviculaire et vers l'épacle. $\grave{A}$ l'examen (Dr. 'Torre, d'Ajaccio) il montre: tuméfaction considérable de la région parotidienne haute surélevant le sterno, torticolis, douleur vive à la palpation on à la pression; pas de rougeur ou d'œdème nets des téguments; pas de trismus; température élevée; voussure de la paroi latérale du pharynx refoulant l'amygdale gauche en avant et en dedans, effaçant le pilier postérieur. Le 11 Décembre, intervention: incision de 5 à $6 \mathrm{~cm}$. sur le bord antérieur du sternocléido-mastoïdien au dessus du tronc thyro-linguo-facial; dès que l'aponévroșe est effondrée, issue de pus fétide; drainage par faisceau de crins. Le 16 Décembre, seconde incision sur le bord postérieur du sterno, avec drainage. Le 18 Décembre apparaît un nedème du bras qui fait craindre une phlébite jugulaire, tronc brachiocephalique; l'œedème a disparu progressivement les jours suivants Le 30 Décembre, il se produit une fistulisation de l'abcès sur la paroi latérale du pharynx. Le 11 de Janvier 1951, en raison d'une fusée purulente dans la loge viscérale au dessus du tronc thyro-linguo-facial, incision de 4 à $5 \mathrm{~cm}$. pour drainer ce clapier. Le 17 Février les plaies étaient guéries.

Depuis le début, le malade présente dysphonie, troubles de la déglutition avec reflux nasal des liquides et accès de toux, dysphagie l'obligeant à ne manger que des aliments hachés. Pendant les premières semaines, des vomissements 
alimentaires surviennent, soit à la fin du repas, soit au plus tard une demiheure après, puis disparaissent.

En Décembre 1951, lorsque le malade nous est envoyé il présente une atleinte des quatre derniers nerfs craniens et du sympathique cervical: paralysie de la moitié gauche du pharynx avec mouvement de rideau; hypoesthésie de la partie postérieure de la langue au tact et à la piqûre; diminution de la perception des substances sapides dans la partie postérieure de l'hémi-langue gauche; paralysie complète de l'hémi-voile gauche, paralysie du récurrent avec voix bitonale (la corde gauche est immobile); anesthésie laryngée; parésie du trapèze gauche; hémiatrophie linguale gauche avec incurvation de la langue vers la gauche et nombreuses contractions fibrillaires; à gauche, léger myosis et discrète enophtalmie. Par ailleurs l'examen neurologique est négatif, ainsi que l'examen général. Les bruits cardiaques sont normaux et réguliers; tension artérielle à 12-7. L'auscultation pulmonaire ne révèle aucun signe anormal, l'abdomen est souple, le foie dans ses limites, la rate ni percutable ni palpable. Enfin de BW est négatif, l'azotémie à $0,35 \mathrm{~g}$., la glycémie à $0,90 \mathrm{~g}$.; les radiographies cervicales et craniennes sont normales et ne montrent, en particulier, aucune anomalie de l'atlas ni de l'axis. Le bilan fonctionnel au moment de cet examen montre nette amélioration puisque les vomissements et les accès de toux à la déglutition ont disparu; il persiste seulement une sensation d'arrêt des solides au niveau de la fourchette sternale.

Deux points méritent, dans ce cas, d'attirer l'attention: l'un, d'un intérêt plutôt doctrinal, le réflexe tussigène provoqué par l'irritation de l'oreille; l'autre, aux conséquences pratiques importantes, la paralysie de l'oesophage.

Notre malade nous a signalé lui-même avoir une quinte de toux chaque fois qu'il provoquait l'irritation de la conque de l'oreille à gauche, et nous avons pu provoquer nous-mêmes ce réflexe. L'exploration nous a montré, d'ailleurs, que ce dernier n'était pas localisé uniquement à cette région, mais intéressait également la paroi antérieure et postérieure du conduit auditif externe, la région rétro-auriculaire jusqu'à la racine des cheveux, l'angle de la mâchoire et la face postérieure de la branche montante du maxillaire inférieur. Ce signe de la toux auriculaire, décrit par Escat, est attribué à l'irritation du filet auriculaire du nerf pneumogastrique. Cette branche naît, au niveau du trou déchirẻ postérieur, du ganglion jugulaire, se porte vers l'aqueduc de Fallope, donne une anastomose au nerf facial, puis s'engage dans un conduit osseux qui l'amène à la base du crâne entre le conduit auditif interne et la mastoïde. Un de ses filets se distribue à la paroi postéro-inférieure du conduit auditif externe et à la face postérieure du pavillon. Chez certains sujets il existe une hyperexcitabilité de cette zone, dont le moindre attouchemeni provoque la toux.

Dans notre cas il existait aussi une sensation de striction pharyngée, peut-être due à une répercussion sur le rameau pharyngé du pneumogastrique. Cependant il nous a semblé que la zone de Ramsay-Hunt, dépendant du nerf de Jacobsen, hranche exocranienne du nerf glosso-pharyngien, était susceptible de provoquer à la fois la toux et cette sensation pharyngée, si bien qu'il est difficile de savoir s'il faut incriminer le IX ou le X paire, peut-être les deux, dans l'apparition de ce symptôme. 
La particularité la plus importante, de notre cas réside dans la paralysie de l'oesophage.

On peut classer les paralysies de l'œsophage en deux grandes catégories: les paralysies œsophagiennes par lésions centrales, encéphaliques ou bulbaires, telles que ramollissement, gomme, hémorragie, traumatismes, tumeurs; les paralysies resophagiennes par lésions périphériques, qui nous intéressent particulièrement ici, et qui peuvent porter sur les noyaux d'origine, sur les nerfs vagues, ou sur l'innervation intrinsèque propre de l'œesophage. Dans ce deuxième groupe, deux affections surtout sont responsables de la paralysie osophagienne: le botulisme et la diphtérie.

Survenant après l'apparition de troubles oculaires à type de paralysie de l'accommodation et des troubles déficitaires frappant les diverses fonctions sécrétoires, la paralysie osophagienne par botulisme peut-être associée à d'autres paralysies intéressant la langue, le pharynx, le tractus gastro-intestinal, la vessie, ou, au contraire, exister seule et dominer manifestement le tableau clinique.

Ces faits, qui paraissaient exceptionnels au moment où Worms et Gand publiaient un cas de paralysie œsophagienne de nature botulinique (Congrès Français d'Oto-Rhino-Laryngologie, 1922), se sont révélés plus fréquents par la suite en raison de la recrudescence du botulisme en France, pendant la guerre (Mounier-Khun, Monıet, Moinecourt et Favre-Gilly, J. de Médicine de Lyon, Sept. 1946).

Collet est un des premiers (Annales de Médicine de l'Oreille, 1931) a avoir décrit une paralysie de l'œsophage au cours d'une paralysie diphtérique unilatérale atteignant les nerfs du trou déchiré postérieur. La pâte barytée restait dans le tiers supérieur du conduit œsophagien anormalement dilaté ou atone, et descendait difficilement dans sa partie inférieure. Ceł auteur signalait, en outre, que l'accumulation de salive dans les sinus piriformes (signe de Chevallier-Jackson) n'existait pas seulement dans les sténoses asophagiennes, mais aussi en cas d'atonie de ce conduit. Ultérieurement Mounier-Khun (Lyon Médical, 7 Août 1938), Mounier-Kuhn et Perreau (J. de Méd. de Lyon, 5 Nov. 1943) et Mounier-Kuhn et Sédaillan (Presse Médicale, 14 Avril 1945) reviennent sur cette question des paralysies de l'œsophage au cours des polynévrites diphtériques. La bouillie pâteuse descend très lentement, met parfois 15 à 40 minutes pour effectuer ce trajet à cause de l'absence de mouvements péristaltiques: l'œsophage reste visible dans toute sa longueur sous forme d'un ruban irrégulier. Si la bouillie est claire, au contraire, la descente est plus rapide que normalement, mais l'œsophage reste longtemps imprégné par la baryte, même si l'on faii avaler de l'eau. La déglutition d'un cachet montre une descente par saccades avec arrêts irréguliers.

Dans le cas de De Luna, Paillas, Robert et Fiastre, une paralysie bilatérale des nerfs bulbaires consécutive à une poliobulbite diphtérique, coexistant d'ailleurs avec une paralysie sérothérapique, s'accompagnait d'une 
paraly'sie œsophagienne, nettement améliorée, comme les autres symptômes, par l'injection de Prostigmine (Revue de Neurologie, Mai 1939). Ewenich (Med. Klin., 25 Oct. 1940), a insisté sur un cas de dilatation idiopathique de l'œsophage consécutive à une paralysie diphtérique de la déglutition.

Avec Jourdan (J. de Méd. de Lyon, 5 Juin 19:15) et dans la thèse de son élève Vialtel, Mounier-Kuhn insiste sur la paralysie concomitante de l'estomac au cours des polynévrites diphtériques. Les malades ont une sensation de réplétion gastrique dès les premières bouchées du repas, n’ont ni éructation ni vomissements. L'estomac se remplit en deux temps: la bouillie opaque se concentre pendant quelques instants dans une cuvette supérieure sous-tubérositaire avec poche à air volumineuse due à la grande quantité d'air dégluti; après cet arrêt, elle passe ensuite progressivement dans la partie verticale de l'estomac et descend en cascade. L'estomac est dilaté, ptosé, son péristaltisme diminué. Sédaillan, Maral, Exbrayat, Gaillard, Trayer et Madone (J. de Méd. de Lyon, 5 Avril 1951) ont insisté sur cette dilatation gastrique d'origine vagale pouvant même faire penser à une dilatation aiguë de l'estomac dans la poliomyélite.

Pareils troubles œesophagiens n'avaient pas échappé aux auteurs qui s'étaient occupé du syndrôme du trou déchiré postérieur. Lhermitte et Kyriaco (Soc. de Neurol. de Paris, 2 Fév. 1926) observèrent un malade pour lequel, à cause des difficultés de la déglutition, on avait porté le diagnostic de néoplasie de l'extrêmité supérieure de l'ossophage ayant déterminé une paralysie du récurrent; or l'œsophagoscopie fut négative, et l'examen neurologique décela une paralysie unilatéra!e des IX, X et XI paires. Lemaître et Gaston (Soc. de Broncho-Oesophagoscopie, 21 Nov. 1933) ont signalé un syndrôme paralytique des quatre derniers nerfs craniens qui avait simulé un rétrecissement de l'œesophage. À la même réunion, Soulas et Aubin ont rapporté des faits analogues soit après diphtérie, soit après poliomyélite.

Une des conséquences les plus importantes de cette paralysie de l'œsophage au cours du syndrôme condylo-déchiré postérieur est le passage as* sez fréquent de la bouillie barytée dans le larynx, la trachée et les bron. ches. Mounier-Kuhn dit avoir vu à l'écran ces organes dessinés comme après une instillation de lipiodol. Peut-être faut-il incriminer un spasme de la bouche csophagienne se surajoutant momentanément à la paralysie, gênant le passage dans le tube digestif et permettant plus facilement le reflux dans le larynx. Ce dernier est particulièrement facilité par l'anesthésie pharyngée et laryngée concomitante et l'absence de réflexe tussigène, même lorsque le corps étranger a pénétré dans les bronches. Au laryngoscope, Mounier-Kuhn a vu la salive et les glaires accumulées atteindre le rebord de la "margelle laryngée" et s'écouler comme un trop-plein dans l'entonnoir laryngo-trachéal. Ce transit anormal de la bouillie barytée peut fort bien se produire pour les aliments déglutis. Cependant il est exceptionnel de voir des complications pulmonaires consécutives à ces paralysies æsophagiennes: à peine Mounier-Kuhn signale-t-il un abcès gan- 
gréneux du poumon droit à évolution favorable, abcès dont il considère l'origine comme peu probante dans un article ultérieur. Il est vrai que, les polynévrites diphtériques ne durant que quelques mois, la paralysie de l'œesophage n'est pas de longue durée.

Chez notre malade, lors a'un examen pratique en Décembre, on constata que la baryte s'accumulait dans le pharynx et ne passait que difficilement dans l'œsophage. Divers clichés montrèrent d'abord une zone d'arrêt haut située dans l'œsophage cervical, dont la limite, concave en bas, dessinait les sinus piriformes. À ce stade, le trajet œsophagien ne se dessinait pas. Par contre une partie de la substance opaque a pénétré dans la trachée et dessinait la bronche gauche et ses ramifications inférieures de la base. Un peu plus tard, on aperçoit l'œesophage qui restait dessiné dans toute sa longueur, nettement dilaté. Enfin lá baryte passa dans l'estomac dilaté qui soulevait nettement le diaphragme par une poche à air très volumineuse. Elle s'arrêtait et s'accumulait au niveau de son tiers supérieur avant de descendre plus bas.

Cet examen n'a été suivi d'aucun phénomène réactionnel bronchique. Par contre nous avons appris du malade qu'il avait présenté il y al quelques mois une "congestion pulmonaire" discrète, qui n'aurait duré que quelques jours et pour laquelle se pose la question de la possibilité d'une pneumonie de déglutition a minima.

Fait important, quand nous avons ultérieurement pratiqué un nouvel examen baryté, la substance opaque est passée directement et facilement dans l'œsophage sans envahissement de l'arbre respiratoire. L'œsophagoscopie n'a pas montré de lésion de l'œesophage en particulier, ni de la bouche œsophagienne, ni des sinus piriformes.

Faculté de Médecine, Marseille, França. 